\title{
MOVILIDAD DEL PUEBLO GITANO DE EUROPA DEL ESTE EN EL ASENTAMIENTO CHABOLISTA DE EL GALLINERO
}

\section{MOBILITY OF THE GYPSY PEOPLE OF EASTERN EUROPE IN THE SHANTY TOWN SETTLEMENT OF EL GALLINERO}

\author{
José David Gutiérrez Sánchez \\ Universidad Pablo de Olavide \\ jdgutsan@upo.es
}

Resumen: El artículo realiza un análisis de la movilidad del colectivo de ciudadanos rumanos de origen romaní en España. Toma como estudio de caso a los romaníes del poblado El Gallinero (Madrid)y destaca la intervención social con menores gitanos rumanos con el fin de establecer estrategias para proyectos de Trabajo Social con dichos colectivos. El trabajo pone de manifiesto cómo los proyectos de intervención llevados a cabo en contextos de riesgo no consiguen prosperar, por una parte, debido a dicha constante movilidad, y por la otra, debido a la escasa implicación política por querer solventar los problemas existentes en dicho poblado.

Palabras Clave: Gitanos, rumanos, movilidad, menores, El Gallinero.
Abstract: The article analyzes the mobility of Romanian citizens of Roma origin in Spain. It takes as a case study the Roma from the village of El Gallinero (Madrid), and emphasizes social intervention with Romanian Roma minors to establish strategies for social work projects with such groups. The work shows how intervention projects done in risk contexts fail to thrive due to 1) constant mobility, and 2) the lack of political involvement to solve existing problems in this town.

Keywords: Gypsies, Romanians, mobility, minors, El Gallinero. 


\section{Introducción}

El Gallinero es considerado por las entidades sociales que operan en el lugar como punto de exclusión social y pobreza (Gutiérrez, 2015). Se tiene en cuenta El Gallinero debido a que hablamos de un poblado que data desde 2001 y es fruto del derribo de las Unidades de Realojamiento Especial de La Quinta, La Celsa, La Rosilla, y sobre todo de las actuaciones desarrolladas para desmantelar el poblado de las Barranquillas llevado a cabo por el Ayuntamiento de Madrid a finales de los años noventa (Gutiérrez, 2017). Por lo tanto podemos decir que debido al peregrinaje provocado por el desmantelamiento de otras zonas en la ciudad de Madrid, El Gallinero forma parte de un producto final dónde muchas familias terminaron asentándose. Además las familias sujeto de esta investigación provienen en su mayoría de Tanderei (Provincia de lalomita), es decir, comparten mismas características y son muchas de ellas las que se conocen entre sí desde origen.

Hablamos de una movilidad de carácter familiar cuyos motivos principales para comenzar dicha acción son generalmente por causas económicas (Gutiérrez, 2015). Dentro de esta investigación, se ha evidenciado cómo el motivo económico del colectivo sujeto de este estudio, afecta a la movilidad familiar por diversos países de Europa (sobre todo por Italia, Francia, Reino Unido, Portugal y España). Dentro del aspecto familiar, señalamos como los gitanos han preferido viajar acompañados fortaleciendo su red de apoyo en el lugar de destino manteniendo tradiciones y cultura, por supuesto relacionándose con la sociedad pero sin desconectar de sus raíces en origen. Massey (1987), Boyd (1989), Fawcett (1989) y Jiménez (2006) señalan como las redes de parentesco y de amistad se muestran básicas en la formación y apoyo dentro de los desplazamientos propios de la migración o movilidad como este caso. Sobre la cultura de las sociedades, esta está compuesta de una serie de elementos precisos, cómo son entre otros, los valores, las ideas, las costumbres, los símbolos, las creencias, etc., y como expresa Herranz (2003: 240), "se disponen a través de la interacción social de los sujetos y emplazan la conducta de éstos en la sociedad".

Este trabajo trata de disertar cómo afecta la movilidad en contextos catalogados como de riesgo al desarrollo de los menores en El Gallinero, así cómo evaluar aquellas intervenciones llevadas a cabo por las administraciones y su impacto en la población. Hay que tener en cuenta que el número de personas que viven en El Gallinero aproximadamente gira en torno a las 400 de las cuales el $50 \%$ son menores de edad. El asentamiento se ubicaba a un kilómetro de la Cañada Real Galiana y presentaba grandes desventajas en materia de acceso a recursos públicos (colegios, farmacias, centros de salud).

La población vive en chabolas de 15-20 metros cuadrados y carecen de letrinas y zonas para cocinar. Según Gutiérrez (2017) el terreno dónde se ubica el poblado presenta irregularidades por lo que el acceso a determinados puntos resulta ser una tarea dificultosa, sobre todo para poder acceder en caso de emergencia (incendios o necesidad de ambulancia).

Hablamos de movilidad y no de migración (Marcu, 2013), ya que desde la incorporación de Rumanía a la Unión Europea sus ciudadanos se convierten en comunitarios con derecho a circulación, es decir, gozan de libertad para "moverse" y no ser tratados como europeos con los obstáculos jurídicos que anteriormente tenían.

La primer parte de este artículo trata la cuestión por medio del análisis de los conceptos que giran en torno a la movilidad y además la metodología empleada en la investigación. A continuación se analiza la movilidad en la infancia y los actores partícipes 
en el asentamiento chabolista de El Gallinero, para finalizar con las conclusiones, las cuales muestran interés en cuanto a la comprensión de los procesos y la implicación de los actores sociales en todo el proceso de intervención social.

\section{Marco teórico: movilidad y desigualdad social}

Desde la entrada de Rumanía a la Unión Europea (2007) se evidencia una atención diferente para referirse a los desplazamientos de dicho colectivo incorporando de tal modo la movilidad, es decir, pasamos de considerar a los rumanos cómo inmigrantes, a reconocer a éstos cómo ciudadanos de pleno derecho en la Unión Europea que tienen capacidad de moverse, ya que es reconocido su estatus cómo ciudadanos de la Unión. Si es cierto y como bien señala Aysa-Lastra y Cachón (2013) las migraciones son en sí mismas procesos de movilidad social, al entenderse como un proyecto transnacional por medio del cual los sujetos pretenden mejorar la posición social tanto individual como colectivamente (Suárez-Grimalt, 2017). La movilidad incorpora elementos que están relacionados con la libertad. Según Bauman (2000: 21) "liberarse, al hablar de emancipación, significa deshacerse de las ataduras que impiden o debilitan el movimiento, es sin más, sentirse libre en cuanto a la capacidad de actuar y por supuesto de moverse".

Según Pajares (2007: 51) "la normativa comunitaria establece para todos los ciudadanos de la Unión, el derecho a la libre circulación de personas y a la libre circulación de trabajadores". Por lo tanto y dentro del contexto que considera este trabajo, las personas que viven en El Gallinero experimentan dos momentos importantes, por un lado el reconocimiento cómo inmigrante hasta 2007 y en segundo lugar cómo ciudadano con derechos desde esa misma fecha.

El caso que nos ocupa además manifiesta otro aspecto significativo, un colectivo que además de ser de otro país, es de etnia gitana. Relacionando la movilidad con el Pueblo Gitano podemos subrayar cómo los gitanos han demostrado a lo largo de la historia que es posible mantener una identidad que no tiene vinculación territorial (Sordé, Flecha y Mircea, 2013). Habermas (2000: 238) señala cómo "se puede compaginar una identidad cultural determinada con la pertenencia a distintas realidades estatales o nacionales". En resumidas cuentas, los gitanos ya han experimentado varios procesos desde sus orígenes, por un lado han vivido la experiencia de la movilidad y por otro el sentir y ser de lugares distintos pero con el reconocimiento de todo un pueblo a nivel global. Según Viruela (2016) tanto en geografía como en sociología, el término de movilidad se emplea esencialmente para referirse al desplazamiento de personas en el espacio y además, ha conocido conceptualizaciones y distintos campos de aplicación (Imbert et al, 2014).

Desde la visión de la sociedad hacia el Pueblo Gitano se evidencian aquellos agentes que forman parte de la construcción social de la movilidad (Gutiérrez, 2017), relacionando todavía hoy día la movilidad con elementos de la teoría migratoria (Marcu, 2013) dónde se combinan conceptos como transnacionalismo o lugar, y en el caso de los gitanos y de origen rumano, flujos migratorios promovidos por la situación carente de empleo en origen. La interpretación de la movilidad no puede llegar a convertirse en un vehículo que ponga en duda constantemente las raíces del sistema social, sino incorporando dicha movilidad se asimilen nuevas formas y constructos que aumenten la esencia cultural y a su vez alimenten la definición de realidades, reconociendo a todas las partes involucradas dentro de una sociedad europea y dinámica cómo la que vivimos. 
Partiendo de la interpretación de movilidad y analizando los contextos de riesgo que soportan las familias en entornos degradados como es el caso de El Gallinero, podemos subrayar que la exclusión social que vive el colectivo es en sí acontecimiento (Vidal, 2009). La exclusión social es algo que está ocurriendo, se muestra dinámica, por lo tanto no es apropiado dentro de la movilidad interpretar lo que acontece como un proceso estático que solo es capaz de intervenirse por medio de algunas vías (o que va y viene a su antojo), sino es necesario estudiar y analizar qué aspectos evolucionan y que afectan al desarrollo y mejora de la calidad de vida de este colectivo. En este sentido el riesgo es un concepto multifacético (Stevenson, 1999; Ryan, 1996; Warner, 1992). Se entiende el riesgo como probabilidad de que se desarrollen determinadas situaciones. Si el hablar del riesgo supone hablar de probabilidades, cuando hablamos de intervención sobre situaciones de riesgo, hablamos de reducir las probabilidades (Vélaz de Medrano, 2002).

La sociedad del riesgo es un concepto abordado ampliamente por Ulrich Beck el cual se basa, como señala Climent (2006), en la constatación de que en las sociedades actuales la producción social de riqueza va acompañada sistemáticamente por una creciente producción social del riesgo. Este punto llevado al proceso de globalización y no solo interpretado desde lo económico, nos facilita la comprensión de cómo las instituciones sociales fracasan a la hora de lograr una estabilidad ante la resolución de problemas concretos que no llegan a intervenirse adecuadamente. Por lo tanto es destacado fijar la atención en la posición que adquiere la política para la resolución y gestión del riesgo (Montenegro, 2005), además de la propia desigualdad social la cual se aprecia como multidimensional, dentro de un contexto móvil y no lineal como es la propia movilidad.

En contextos chabolistas como el de El Gallinero, la desigualdad social puede superarse por medio de la integración social de las familias. El concepto de integración social según Subirats (2005) ha estado tradicionalmente asociado con la inserción al mercado laboral, considerando de tal modo el empleo como el principal mecanismo de integración social (Epelde, 2017). Sin embargo, ante la dificultad actual por obtener un empleo debido mayormente por la crisis económica que afecta Europa, es pertinente estudiar otros elementos que puedan tener cierto potencial integrador y puedan a su vez suplir las carencias de los mecanismos tradicionales. La apuesta por el desarrollo y creación de proyectos de intervención social que incidan en determinados aspectos que las administraciones públicas no puedan acceder, resulta importante su consideración. De este modo, se plantea en este trabajo que más allá de factores clásicos como el acceso al mercado laboral o la formación, la integración social debe tener en consideración la movilidad del colectivo en cuestión, debido mayormente a que puede afectar en la estabilidad de dichas intervenciones y además incidir negativamente en aquellas personas más vulnerables, los niños (Gutiérrez, 2017).

\section{Metodología}

Los datos que aquí se exponen forman parte de los resultados de un proceso de investigación mediante la aplicación de técnicas y métodos cualitativos de perfil socioetnográfico en el asentamiento chabolista de El Gallinero (Madrid), durante el periodo de tiempo entre 2010-2014. El objetivo central de la investigación era: realizar una investigación evaluativa de la intervención social con menores gitanos rumanos con el fin de establecer estrategias para proyectos de Trabajo Social con dichos colectivos. 
En esta investigación se utilizaron tres técnicas diferentes para la obtención de la información; entrevistas en profundidad, Investigación-Acción Participativa (en adelante IAP) y observación participante por medio de la incorporación en las diversas actividades (trabajo de campo) que la Fundación Imaginario Social llevaba a cabo durante el periodo de tiempo entre 2010-2014 en dicho asentamiento.

En primer lugar se llevaron a cabo un total 86 entrevistas clasificadas de la siguiente forma: 9 entrevistas realizadas a miembros de entidades sociales (ONG, Fundaciones, Asociaciones, Parroquias), 11 efectuadas por medio del Proyecto PUCAFREU (Promoting unprotected unaccompained Children 's Access to fundamental rights in the European Union) de CNRS-Migrinter, 8 a informantes clave o líderes, 22 a familiares de los sujetos de la intervención, 31 a menores entre 10 y 18 años, y 5 entrevistas realizadas grupalmente por medio de actividades en el asentamiento de 2010 a 2012. El propósito de las entrevistas en este estudio fue que cada entrevistado proporcionara una información específica de las intervenciones sociales de las que ellos participaban y además, en el caso de los menores, analizar qué aspectos afectan al desarrollo de los mismos y conocer las causas del porqué determinados proyectos no terminan estableciéndose en el asentamiento.

Nuestra investigación se trata de un estudio etnográfico en el que se emplea el estudio instrumental de casos con análisis cualitativo (Stake, 1998). Partiendo de ello, a la hora de estudiar por medio de las técnicas de investigación los sujetos, se encontraron ciertas limitaciones en la recogida de la información, por ejemplo, casos de hermetismo por parte de la comunidad asentada, es decir, ante la elaboración de una entrevista muchas personas no eran capaces de argumentar aspectos culturales o los medios de subsistencia que empleaban, por lo cual se tuvieron que realizar las entrevistas en muchos casos con juegos como teatro y role-playing. Por otro lado y sobre todo ante preguntas relacionadas con los medios de subsistencia, los entrevistados tenían cierto miedo de contestar por presión grupal, por lo que algunas entrevistas se llevaron a cabo fuera del asentamiento. Dicha presión era motivada mayormente a la relación en la que se emplaza al colectivo con el presunto robo de cobre y delincuencia. "La cuestión de fiabilidad y validez de los estudios cualitativos es algo complejo de asegurar" (Pozuelos, Rodríguez y Travé, 2012: 568) por ello recurrimos al principio de credibilidad próximo al criterio de calidad para las narrativas de investigación-acción de Heikkinen, Huttunen y Syrjälä (2005). Apelamos a dicho principio debido a que en cuantiosas ocasiones detectamos que diferentes sujetos reproducían hechos de formas dispares, forzándonos a salir del asentamiento en determinados casos para extraer toda la sinceridad que en diversas ocasiones por presión del grupo no son fáciles de argumentar.

Con respecto a las técnicas de IAP, éstas se llevaron a cabo por medio del Proyecto Nido y Proyecto EducaRom en el asentamiento para poder analizar la realidad social de la comunidad asentada e indagar sobre aquellos procesos participativos sociales dentro del grupo de jóvenes y la comunidad (Valderrama, 2013). Ambos proyectos tenían como objetivos centrales la intervención socioeducativa de jóvenes menores de edad por medio de actividades de educación no formal. En este sentido la IAP según Fals-Borda (1985) se trata de la inserción del investigador en la comunidad, analizando las condiciones históricas y estructura social, estudiando el nivel de desarrollo de conciencia de los miembros de dicha comunidad en relación a los problemas focalizados, el desarrollo de las organizaciones políticas y grupos de acción y el compromiso a la solución de problemas con la comunidad o grupo (Balcazar, 2003). Según Selener (1997) el grado de participación de los miembros de la comunidad o grupo determina el nivela de IAP. 
Para llevar a cabo la IAP nos hemos decantado por las tres actividades centrales que Balcazar (2003) señala dentro de la misma;

- En primer lugar investigación: análisis funcional de antecedentes y consecuencias, además de identificar las necesidades (Fawcett, Seekins, Whang, Muiu y Suárez-Balcazar, 1982). Una vez realizado el análisis y la identificación de necesidades, los participantes (sujetos del estudio) determinan las prioridades y se organizan grupos de acción para planear con carácter sistémico el proceso de intervención para la solución de los problemas focalizados.

- Educación: "los participantes aprenden a desarrollar una conciencia crítica la cual les permite obtener las causas de sus problemas y se identifican potenciales soluciones" (Fleitas y Molero 2015:205). Según el autor se trata sencillamente de enseñar a la gente a descubrir su propio potencial para actuar. En el caso específico de nuestra investigación no se trataba solamente de identificar las potenciales soluciones tomando como base el aspecto económico (como carencia de bienes materiales) sino comprender las necesidades como potenciales (Krmpotic, 1999; Jiménez, Gutiérrez y Diz 2014).

- Por último, los participantes implementan soluciones prácticas a sus problemas utilizando sus propios recursos, o aquellos obtenidos por medio de la solidaridad de otros grupos o entidades. Estas actividades están interconectadas y forman un ciclo dinámico.

Tanto la observación participante como la IAP eran especialmente atrayentes para el estudio de la vida cotidiana tanto de la propia comunidad asentada como de todas aquellas instituciones y colectivos que tenían una tarea específica en el poblado.

La participación directa con los sujetos de este estudio posibilita indagar sobre los procesos de exclusión social que se desarrollan en lugares periféricos de la sociedad, además de centrarnos en la consecución de los objetivos dentro de la investigación. En este sentido Shaw (1999) argumenta lo siguiente; "Debido a que la epistemología subjetiva del paradigma de la investigación cualitativa ve la realidad social como algo construido por las personas, el investigador no puede permanecer distante del fenómeno social en el cual está interesado". Es por ello que ante un colectivo tan alejado por su ubicación y desconocimiento de cultura por parte de la sociedad en general (Vergnano, 2014), la aproximación se muestra necesaria para la comprensión de la perspectiva de los participantes dentro de la realidad social en la que están inmersos (Callejo, 2002; Martínez, 2006).

La investigación está ubicada en contextos catalogados como de riesgo. Desde un punto de vista sociológico en relación a los niños y adolescentes, comprendemos su lugar en el seno de las diferentes configuraciones de relaciones de interdependencia entre los actores que componen la familia, grupo de iguales y la institución escolar (Lahire 2007).

Los llamados contextos de riesgos para la infancia, originan en la mayoría de los casos, para los niños y las niñas que lo sufren, procesos traumáticos complejos y acumulativos (Barudy 2014), por supuesto también dentro de la movilidad a sus familias. Estos contextos muestran la incapacidad del adulto para brindar alternativas completas y globales de intervención hacia los niños y niñas, y además, cómo dichos escenarios juegan un papel trascendental en el mantenimiento del bienestar y oportunidades. 


\section{Movilidad de la infancia: implicaciones sociales y adaptabilidad}

"No sé si acabaré aquí. España está muy bien pero El Gallinero es una mierda.

Iré dónde pueda conseguir dinero, pero por ahora me quedo aquí".

Entrevista a C.I. (2012). Chico de 17 años.

A lo largo de nuestra investigación, nos hemos aproximado a aquellos procesos que evidencian los menores de edad dentro de la movilidad que caracteriza al colectivo gitano rumano. Según las entidades sociales que operan en el asentamiento chabolista de El Gallinero, aproximadamente en la actualidad viven unas 400 personas, de las cuales el $50 \%$ son menores de edad.

En el Gráfico 1, podemos apreciar la evolución del número de familias asentadas en El Gallinero entre 2008-2016. Es el año 2011 cuando más personas viven en el poblado, pero a su vez es el año junto con 2012 que más redadas se llevan a cabo, con su impacto en la disminución de personas y movilidad total de las familias. El Gallinero es un poblado chabolista asentado en un solar de propiedad privada ubicado en la periferia de la ciudad de Madrid (Gutiérrez, 2015) y dónde las familias comenzaron a construir sus hogares sin permisos por parte de la administración local. Este problema de ocupación unido a las detenciones de varias personas por supuestos robos de cobre en fábricas y líneas de trenes, provocaron en 2011 que se intensificaran las redadas.

Gráfico 1: Número de familias por año

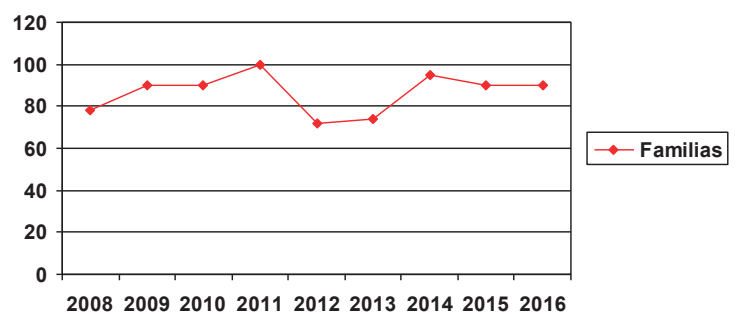

Fuente: Elaboración propia. Datos de ONG y Parroquias

Tabla 1: Familias y población total aproximada

\begin{tabular}{|c|c|c|}
\hline Año & No Familias & Población \\
\hline 2008 & 78 & 386 \\
\hline 2009 & 90 & 440 \\
\hline 2010 & 90 & 404 \\
\hline 2011 & 100 & 600 \\
\hline 2012 & 72 & 321 \\
\hline 2013 & 74 & 390 \\
\hline 2014 & 95 & 435 \\
\hline 2015 & 94 & 400 \\
\hline 2016 & 90 & 400 \\
\hline
\end{tabular}

Fuente: Elaboración propia. Datos de ONG y Parroquias 
En la tabla 1 podemos apreciar la relación entre familias y población. Destacamos la alta natalidad registrada en el asentamiento siendo el número de niños y niñas aproximadamente la mitad del total según las entidades sociales que operan en el asentamiento (Gutiérrez, 2015).

El alto número de niños está directamente relacionado con la temprana formación de una familia, ya que cómo hemos comprobado en el asentamiento la edad media de casamiento gira en torno a los 13 años (cuando la madurez sexual de las chicas es normalmente evidenciada) pero por supuesto existen casos comprobados de matrimonios a edades más avanzadas. Según Griffin (2008: 258) "el casamiento en edades tempranas permite, para los gitanos, poder concebir hijos a los que se les brinda una mayor fortaleza que a edades más avanzadas".

En nuestra investigación evidenciamos cómo el proceso de movilidad afecta directamente al desarrollo de los menores, es decir, cómo la falta de estabilidad y ubicación en un lugar con todo lo que ello implica (adaptabilidad escolar, relación con iguales, duelos internos, etc.) unido a la inmersión en un contexto catalogado como de riesgo como son los asentamientos chabolistas, ocasiona que las exigencias comunitarias y familiares, incidan en el carácter individual transformando más rápidamente el desarrollo y como consecuencia se lleven a cabo actividades que podemos entender dentro de una sociedad occidental como de adultos o de edades más adelantadas.

En nuestro caso la movilidad está acompañada de la ubicación o asentamiento temporal en lugares periféricos con cierto grado de segregación y exclusión social. Según Vergnano (2014: 90) "los romaníes son identificados por los demás ciudadanos como portadores de degradación y, además, como una amenaza a las propias condiciones de vida ya establecidas en los barrios". Esta consideración puede estar relacionada con los medios empleados por la población sujeto de este estudio para mejorar su calidad de vida. En este sentido por medio de la IAP tenida en cuenta en esta investigación descubriros patrones que son a su vez reproducidos en otros lugares de Europa como Burdeos en Francia (Gutiérrez, 2017), Turín en Italia (Vergnano, 2014) e incluso en otros lugares de España como Barcelona (López y Aharchi, 2012).

La participación de los menores en los procesos de movilidad familiares no es fruto del consenso familiar, sino más bien una necesidad por cubrir y que tiene que abordarse simplemente, por tanto el ejercicio de derecho de opinión en la mayoría de los casos está supeditado a la voluntad adulta, óptica adultocéntrica.

La movilidad se contempla como movilidad laboral, fruto del aumento del desempleo en origen. El conjunto de trabajos destacados y llevados a cabo por el colectivo se ubica dentro de lo que conocemos como economía sumergida. Los empleos más repetidos son la recogida de chatarra (en niños acompañados de sus padres) y la mendicidad en niñas, además de ser éstas quiénes se encargan de realizar las tareas del hogar. Este último hecho sobre las niñas, es abordado por Torres (2013: 160), el cual subraya cómo por lo general, "las mujeres gitanas de estatus sociales medio-bajo e inferior se dedican a trabajar con el marido u otros parientes cercanos" (de ahí ésta marcada movilidad familiar de carácter económico).

En relación a los niños y niñas, que exista una determinada necesidad familiar para que éstos colaboren en la mejora de la calidad de vida grupal, se manifiesta una relación directa entre el nivel de ingresos de los padres con la capacidad de integración en la sociedad. San Román (2006: 145) argumenta cómo existe una "correspondencia entre el nivel de ingresos y la adaptación escolar". Al haber niveles económicos bajos, se pierde en cierta manera las expectativas sociales sobre la escuela 
(por lo general) ya que al estar inmersos en procesos de integración y en continua búsqueda de recursos para sostener el grupo familiar, se muestra más complejo el participar dentro de la estructura social. Una vez llevado a cabo este proceso, resulta posteriormente complejo dinamizar de nuevo la relación con la escuela. A ello le añadimos la movilidad constante del grupo sujeto de este estudio y las condiciones de habitabilidad dentro de los asentamientos, lo cual a su vez incide en la posibilidad de acabar los deberes encomendamos por la escuela, o bien disfrutar de algún modo de las ventajas de estar más próximo a compañeros de clase o con el maestro, ya que hablamos de un poblado ubicado en la periferia de la ciudad de Madrid.

El análisis de las entrevistas, al tratar el sistema de relaciones entre sociedades en la población sujeto de estudio y los procesos que se desarrollan internamente, podemos observar diversas cuestiones:

En primer lugar el proceso mental que afecta a las propias personas, debido mayormente por el incumplimiento de las expectativas depositadas en la movilidad, y cómo ello incide en su comportamiento dentro de la comunidad cómo con la sociedad en su conjunto. Según Kohli (2014: 33) "el movimiento a lo largo del tiempo y a través de los territorios guarda relación con la madurez en la persona". Nos referimos al proceso que vive la persona interiormente (o el colectivo) en su proceso móvil en relación al aprendizaje vivido (tanto por causas positivas como negativas) que hace que articule un bagaje que evidentemente afecta en su comportamiento (presente y futuro) y que posteriormente exterioriza y se hace palpable con la sociedad (provocando estados o momentos que pueden ser interpretados como aceptados o no socialmente).

En segundo lugar, dentro del imaginario del inmigrante, se ubica mentalmente dos espacios, uno en su país de origen y otro en el lugar donde habita durante el tránsito (Marcu, 2013). Podemos reflejar por tanto, como la persona siente una dicotomía interna entre el sentimiento de pertenencia a una sociedad y la necesidad de sentirse parte de la sociedad en la que se establece, aunque cómo señala Fuentes-Gutiérrez (2014) los/as (in) migrantes se hallan influenciados por diversos contextos y esto hace que su sentido de pertenencia no se ubique en un lugar únicamente, sino que, en ocasiones la identificación con el contexto de origen o el de destino se origina de forma paralela. El autor diserta sobre inmigración, pero este caso es reproducido por los sujetos de este estudio aunque ya sean miembros comunitarios de la Unión Europea.

Por otro lado, la unión con el origen puede estar enlazada con los duelos producidos dentro de la movilidad. Identificamos duelo como el proceso de adaptación emocional que sigue a cualquier pérdida y que es significativo para el sujeto (Konrad y Santoja, 2005). Dentro de nuestra investigación señalamos como duelos internos de la persona los siguientes; duelo por la familia y amigos (nostalgia), por la lengua (idioma), por la cultura (tradiciones), por la tierra, por el estatus, y por el contacto con el grupo étnico.

Por último, señalamos que la ubicación del asentamiento (a las afueras de la ciudad de Madrid) impide en numerosas ocasiones poder establecer relaciones de convivencia con la sociedad en general, por lo que se reduce la probabilidad de constituir uniones significativas que puedan situar a los habitantes de El Gallinero en mejores posiciones a la hora de hallar un trabajo, y normalizar la identificación que la sociedad elabora ante personas de distinta etnia (eliminar prejuicios). El emplazar el asentamiento en lugares distantes como las periferias de las ciudades, fragmenta de algún modo las posibles relaciones que puedan establecerse, ya que no se llevan a cabo relaciones entre grupos sino solo con una misma comunidad. Hablamos por 
tanto de "territorios sensibles" (Rojo, 2010: 16), los cuales albergan características particulares como; alto paro juvenil y relaciones frágiles de empleo; fracaso económico; y están estigmatizados por agrupar mayoritariamente a poblaciones económicamente delicadas.

\section{Relaciones entre actores participantes en El Gallinero}

"Ya no voy al Colegio, soy mayor para ir. Mis padres prefieren que me quede aquí y haga las tareas del hogar".

Entrevista a L.D. (2012). Chica de 14 años.

"Quiero una casa mejor y dinero para comer porque mi madre sale a pedir al centro de Madrid todas las mañanas".

Entrevista a M.C. (2012). Chico de 15 años.

Según Lacomba (2008) se desarrollan sistemas de relaciones entre la población de origen y de llegada en un territorio concreto. Estas relaciones pueden de ser de tres tipos; convergentes, divergentes y paralelas. Las primeras se originan cuando coinciden las expectativas entre la población recién llegada y la población que recibe (no se establecen tensiones y se llegan a puntos de unión y encuentro para ambas poblaciones, independientemente de su cultura o tradiciones). Las relaciones divergentes, cuando las necesidades son opuestas y aparecen tensiones entre comunidades inmigradas recién llegadas y comunidades de acogida con la competencia en el mercado laboral o diferencias entre las formulas de integración, más común en momentos de crisis económicas y sociales (Gutiérrez, 2017). Y relaciones paralelas; la movilidad permite un sistema dual, pero no existe una interrelación entre comunidades de origen y comunidades de destino.

Partiendo de estos sistemas de relaciones y dentro de la movilidad que caracteriza al colectivo sujeto de este estudio, podemos plasmar la presencia o la continuidad de relaciones entre la población ubicada en El Gallinero y la sociedad en general. Dichas relaciones son protagonizadas por diversos actores sociales. Entendemos por actores sociales como bien expresa Matus (1987: 53) indicando que éstos son: "motores de cambio, como productores de eventos sociales, y protagonistas del cambio situacional”. Chiara y Di Virgilio (2009) señalan que los actores sociales constituyen "formas concretas", esto es, organizaciones e individuos con capacidad de ejercer incidencia en cuanto dialécticamente relacionados con múltiples campos de acción (Cañizares, 2013).

En El Gallinero existen diversos actores participantes; actores públicos y regionales, actores públicos locales y actores sociales de intervención. Cada participante asume unas tareas específicas aunque en la práctica, las distintas intervenciones pueden plantear ciertas dudas en cuanto su capacidad resolutiva. 
Figura 1: Actores en El Gallinero

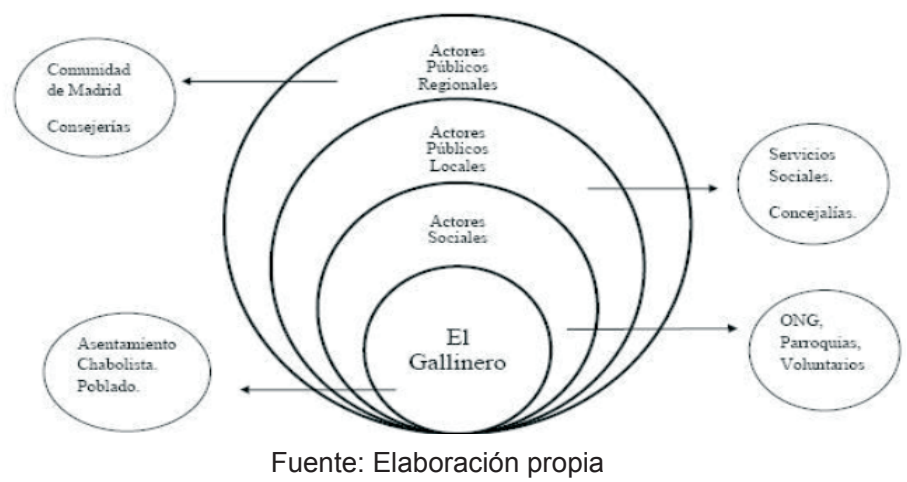

A continuación vamos a detallar el trabajo llevado por cada uno de dichos actores y su impacto en la población:

Actores Públicos regionales y locales: cómo indica la figura 1 se trata de la Comunidad de Madrid por medio de sus Consejerías y el Ayuntamiento de Madrid a través de Concejalías. En el caso que nos ocupa éstos actores durante el periodo de la investigación se ocupaban sobre todo de aspectos relacionados con vivienda y educación. En relación a la primera podemos argumentar cómo se establecían relaciones divergentes y en determinados momentos paralelas debidas mayormente a las políticas de reinserción social y desalojo de la población en el asentamiento. Hablamos de una población que no quiere abandonar el lugar donde se ubica el poblado pero que sin embargo quiere mejorar las condiciones de este. Esto genera discrepancias y durante los años 2010-2012 se llevaron a cabo continuos desalojos y derribos de chabolas. Esto unido directamente con la falta de alternativas por parte de las administraciones en brindar espacios o viviendas para poder vivir mientras se mejora la situación, provocó la vulneración de diversas normas. Según Gutiérrez (2015) y dentro de esta circunstancia resaltamos la Resolución B7-0500 del Parlamento Europeo, sobre la situación del pueblo romaní en Europa, de 7 septiembre 2010, en la cual se habla del derecho a una vivienda digna y la obligación de proporcionar un alojamiento alternativo adecuado, aunque, como pudimos justificar durante nuestro estudio, en el asentamiento se vulnera constantemente. Enlazado con esta resolución, añadimos la vulneración de artículos de la Convención sobre los Derechos del Niño, como son: el Art. 16.1; referido al derecho a la intimidad familiar; el Art. 27; que proclama el derecho a un nivel de vida adecuado y a la protección social en especial respecto a nutrición, vestuario y vivienda; y el Art. 28; que defiende el derecho a la educación y al juego. Aunque estos artículos comprenden el amparo en diversas materias del derecho, la vulneración de los mismos es verificada por entidades sociales que trabajan in situ.

Además si comparamos la situación de El Gallinero con otros puntos de Europa como es Burdeos (Francia) existe una falta de apoyo en el suministro de materiales de construcción para mejorar las condiciones de vida por parte de la administración local, reflejando un escenario de chapa, maderas, plásticos, cartones, etc., dónde se originan plagas de roedores y la acumulación de basuras hace insalubre el lugar. En Burdeos sin embargo se atiende a la población dentro de su contexto de riesgo y movilidad proporcionando ayudas para construcción de casas prefabricadas de madera y el apoyo con letrinas y electricidad (Gutiérrez, 2017). 
Este aspecto incide en la movilidad constante de las familias, las cuales se ven en muchas ocasiones obligadas a seguir móviles debido a la poca capacidad de las administraciones en dotación de personal humano y material.

En materia de educación, las administraciones por medio de sus órganos de gestión (Consejerías y Concejalías) establecieron una metodología de intervención apoyada en Cruz Roja, la cual a través del proyecto Henry Dunant trataba de empoderar y dotar de autonomía a las personas del poblado y además proporcionar la plena escolarización de los niños y niñas. Este caso concreto de la educación obtuvo grandes resultados ya que se pudo escolarizar a casi todos los menores que vivían en el asentamiento, sin embargo, la falta de inversión de recursos por parte de las administraciones y la incidencia a partir de los 13 años aproximadamente, provocaba que con el tiempo los niveles de abandono escolar se intensificaran. Cómo señalamos anteriormente es en esta edad cuando comienzan los casamientos y se exige por parte de la familia y el poblado, mayor implicación en la mejora y dotación de recursos al asentamiento.

La Universidad Pontificia Comillas y Save the Children (2014) coindicen en que El Gallinero se encontraba en una situación de gran aislamiento geográfico e indeterminación administrativa, de tal forma que para poder llegar a la zona de servicios más próxima se debían invertir aproximadamente unos 35 minutos andando campo a través o en transporte público, lo cual incidía negativamente en la asistencia a la escuela y además durante el año 2011 se registraron numerosos incidentes por falta de autobuses que hicieran la ruta entre el asentamiento y el Colegio.

En relación a los actores sociales (ONG, Parroquias y Voluntarios) eran éstos los que persistían en la escolarización de los menores y trabajaban con las familias la importancia de la educación como respuesta a los problemas relacionados con la pobreza. En muchos casos eran los profesionales de estas entidades los que establecían contacto con los institutos y colegios, haciendo hincapié en la necesidad de integrar socialmente a los menores escolarizados. Esto demostraba el gran tejido social que se reproducía en dicha comunidad entre las entidades y el colectivo de estudio. Entre estos actores sociales podemos destacar lo siguiente:

a) Comprobamos que el empoderamiento social entre entidades, con proyectos realizados en común, reducen costes y optimizan recursos, por lo que se pueden mejorar y reforzar otras áreas que puedan estar menos desarrolladas a lo largo de la intervención, además de reducir duplicidades (Barudy, 2005; Gutiérrez y Diz, 2017).

b) La intervención social realizada por medio de dichas entidades y de forma específica sobre problemas concretos (vivienda, salud y educación) establecía una estrategia de acción controlada y coordinada, pero sin embargo, la capacidad de las propias entidades en relación a recursos públicos disponibles era limitada, por lo que las intervenciones en algunos casos eran superficiales y no resolvían a lo largo de los años problemas concretos como por ejemplo aquellos orientados a la educación formal o no formal.

c) Se mostró relevante el que los proyectos pudiesen tener profesionales nativos o con gran experiencia en la materia (cultura gitana, idioma o la vivencia del proceso de movilidad del colectivo) ya que servía como forma de unión entre las personas del asentamiento y generaba mayor implicación social por querer solventar los problemas focalizados. 
d) Los proyectos llevados a cabo por las entidades sociales se veían mermados por la gran movilidad del colectivo, lo cual se transformaba en poca asistencia a las actividades que las entidades desarrollaban. Además la incidencia de las administraciones por medio de desalojos y derribos de chabolas sin previo aviso provocaba que las actividades que se desarrollaban en esos momentos concretos tuvieran que paralizarse y no pudieran mantenerse hasta pasado varios días, debido al nerviosismo provocado por la situación, sobre todo en el caso de arrestos o multas.

Por lo tanto podemos reflejar que a pesar de los escasos recursos de las entidades sociales que operaban constantemente en El Gallinero, éstas obtenían mejores resultados que aquellos gestados por las administraciones. La movilidad del colectivo en este caso sufría alteraciones, es decir, por un lado la intervención social de la administración local y regional provocaba mayor movilidad del colectivo por medio de acciones que se materializaban en presión y desalojos. Del otro lado las intervenciones propiciadas por las entidades sociales permitían que el colectivo se asentara y pudiera mejorar en la consecución de sus objetivos o simplemente salir de la pobreza. Es decir, la presión ejercida por la administración estaba orientada en la ocultación del problema, de ahí ese "peregrinaje" por diversas zonas de Madrid durante años, y por otro lado las entidades sociales permitían dotar de mayor cobertura en relación a mejoras sustanciales en materia de salud, vivienda y educación.

\section{Conclusiones}

Después del recorrido llevado a cabo por los aspectos en torno a la movilidad del colectivo gitano rumano y específicamente en el poblado chabolista de El Gallinero, se puede confirmar la tesis planteada por la investigación en tanto se determina que ciertamente se presentan dificultades en el grupo sujeto del estudio en cuanto a la participación social en las estructuras del Estado, debido mayormente por el carácter errante del pueblo gitano y los procesos de exclusión social que los han acompañado dentro de su propia movilidad entre países. Se resalta las implicaciones dentro de la movilidad en la infancia, evidenciando cómo se trata de una migración familiar, pero que sin embargo no tiene en cuenta la opinión de los niños y niñas en la toma de decisiones en relación a qué país dirigirse y si desean seguir acudiendo a la escuela. La movilidad del colectivo, dentro de la política europea de circulación de personas, expresa desavenencias en relación a la escolarización de los menores. Esto se manifiesta en mayor absentismo y desadaptación escolar, por lo que establecer acciones que posibiliten mejoras para el colectivo resulta tarea compleja para los Estados.

La investigación llevada a cabo en El Gallinero nos muestra como el motivo de la movilidad es económico o laboral. Es imprescindible reconstruir las etapas laborales previas, así como indagar sobre la incidencia de los procesos políticos y estructurales en el país de origen hacia el colectivo gitano de tal forma que pueda interpretarse aquellos aspectos que inciden sobre la decisión final para comenzar el proceso de movilidad. Por otro lado es interesante abordar el perfil de cualificación de los inmigrantes y su capacidad para poner en marcha sus capacidades en otros países con pocos recursos económicos, comprobando las redes de apoyo establecidas dentro del colectivo, las cuales pueden permitir un mejor acceso a un empleo, aprender el idioma con más rapidez y conocer los aspectos administrativos para regularizar la estancia en un lugar en concreto (adquirir la tarjeta sanitaria, inscribirse como demandante de empleo en los sistemas o servicios de empleo regionales, adquirir 
conocimientos sobre los medios de transportes, informarse sobre apoyo asociativo de otros compatriotas para obtener información sobre derechos, etc.).

Por último habría que abordar el papel del Estado en relación a qué medidas se adoptan en la intervención con familias de gran movilidad, es decir, qué planes se ponen en marcha para afrontar el establecimiento de dichas familias en entornos desfavorecidos y, sobre todo, cómo éstos espacios afectan a los menores de edad en cuanto su posición en la sociedad como sujetos a proteger. Habría por tanto que estudiar cuáles son los procedimientos habituales dentro de las Comunidades Autónomas y qué respuestas se ofrecen no solo con el colectivo gitano procedente de Rumanía, sino con otras nacionalidades que llegan a nuestro país. Además sería adecuado analizar el papel de las organizaciones no gubernamentales en espacios denominados como de riesgo (asentamientos chabolistas) y su trabajo en red con las administraciones y otras entidades sociales, de tal forma que pueda interpretarse que aspectos tener en cuenta y que beneficiarían a las familias, e igualmente situar unos mínimos estables y coordinados para la intervención social con menores.

\section{Bibliografía}

Aysa-Lastra, M. y Cachón, L. (2013). Movilidad ocupacional segmentada: el caso de los inmigrantes no comunitarios en España. REIS, Revista Española de Investigaciones Sociológicas, № 144, pp. 23-47. https://doi.org/10.5477/cis/reis.144.23

Balcazar, F.E. (2003). Investigación acción-participativa (IAP): Aspectos conceptuales y dificultades de implementación. Revista Fundamentos en Humanidades. Año IV-Nㅇ/ II (7/8), pp. 59-77.

Barudy, J. 2014. Infancia en contextos de riesgo, traumas infantiles y resiliencia. En Jiménez, A.S., Pantoja, A., Leiva, J.J. y Moreno, E. (Coords.). Infancia en contextos de riesgo. XXV Años de la Convención sobre los Derechos del Niño. Granada: GEU Editorial.

Bauman, Z. (2000). Modernidad líquida. Argentina: Fondo de Cultura Económica.

Beck, U. (1992). Risk society. Londres: SAGE Publications. https://doi. org/10.4135/9781412952552.n244

Boyd, R.D. (1989). Facilitating Personal Transformations in Small Groups: Part I. Small Group Behabiour, No 4, pp. 459-474. https://doi.org/10.4324/9780203359099_chapter_eight

Callejo, J. (2002). Observación, entrevista y grupo de discusión: el silencio de tres prácticas de investigación. Revista española de Salud Pública, № 76, pp. 409-422. https:// doi.org/10.1590/s1135-57272002000500004

Cañizares, B.Z. (2013). Actores Sociales: claves analíticas aproximación problematizadora. Centro de Estudios Interdisciplinarios Problemáticas Internacionales y locales, CEIPIL. Tandil.

Chiara, M. y Di Virgilio, M. (2009). Gestión de la Política Social. Conceptos y Herramientas. Buenos Aires: Editorial UNGS/Prometeo.

Climent, V. (2006). Sociedad del riesgo: producción y sostenibilidad. Revista Papers, $\mathrm{N}^{\circ}$ 82, pp. 121-140. https://doi.org/10.5565/rev/papers.2052

Epelde, M. (2017). Proyectos de apoyo social como herramienta de integración social de los jóvenes inmigrantes no acompañados. El Proyecto IZEBA de Gipuzkoa. En 
Jiménez, A.S. et al. (Coord.). Reconstruyendo un mundo con ojos de niñas. Entre la pobreza y la educación. Granada: GEU Editorial.

Fals Borda, O. (1985). Conocimiento y poder popular. Bogotá: Siglo XXI.

Fawcett, J.T. (1989). Networks, linkages and migration systems. International Migration Review, № 23 (3), pp. 671-80. https://doi.org/10.2307/2546434

Fawcett, S.B., Seekins, T., Whang, P., Muiu, C. y Suárez-Balcazar, Y. (1982). Involving consumers in decision-making. Social Policy, № 13 (6), pp. 36-41.

Fleitas, R. y Molero, N. (2015). La investigación acción participativa en procesos de desarrollo comunitario: una experiencia de cooperación interuniversitaria en el barrio de Jesús maría, La Habana Vieja (Cuba). Pedagogía Social. Revista Interuniversitaria, № 26, pp. 203-228. https://doi.org/10.7179/psri_2015.26.08

Fuentes-Gutiérrez, V.M. (2014). Intervención social local con mirada global. La propuesta de trabajo social transnacional con familias (in) migrantes y/o transnacionales entre Bolivia y España. Portularia, Vol. XIV, N¹, pp. 87-95. https://doi.org/10.5218/ prts.2014.0008

Griffin, C. (2008). Nomads under the Westway. Irish Travelers, Gypsies and other traders in west London. UK: University of Hertfordshire Press.

Gutiérrez, J.D. (2017). Imaginarios sociales de la itinerancia dentro de la diáspora gitana. Revista Interdisciplinaria sobre Imaginarios Sociales, Imagonautas. № 9, pp. 40-57.

Gutiérrez, J.D. (2017). Menores gitanos rumanos en Madrid y Burdeos: entre exclusión y pobreza. En Jiménez, A.S. et al. (Coord.). Reconstruyendo un mundo con ojos de niñas. Entre la pobreza y la educación. Granada: GEU Editorial.

Gutiérrez, J.D. y Diz. J. (2017). Cooperación Internacional en Marruecos: empoderamiento para insertar socio-laboralmente a jóvenes en contextos de riesgo. Revista Cuadernos de Trabajo Social, № 30 (1), pp. 163-174. http://dx.doi.org/10.5209/CUTS.50352

Gutiérrez, J.D. (2015). Los menores gitanos rumanos de "El Gallinero": Etapas de desarrollo en un contexto de riesgo. Revista Electrónica de Investigación y Docencia (REID), No 13, pp. 27-44.

Habermas, J. (2000). The inclusión of the other. Studies in Political Theory. Cambridge Mass: MIT Prees.

Heikkinen, H.L.T., Huttunen, R. y Syrjälä, L. (2005).On the problem of quality in narratives of action research. Ponencia en la Conferencia anual de la European Educational Research Association. Dublín.

Herranz, G. (2003). Sociología y delincuencia. Granada: Editorial Alhulia.

Imbert, C., Duducs, H., Dureau, F. y Giraud, M. (2014). D'une métropole á l'autre. Pratiques urbaines et circulations dans l'espace européen. París: Armand Colin.

Jiménez, A. S., Gutiérrez, J. D. y Diz, J. 2014. Infancia, Cultura y Emoción. Un Escenario Internacional de Formación. En Jiménez, A. S., Pantoja, A., Leiva, J. J. y Moreno, E. (Coords) Infancia en Contextos de Riesgo: XXV Años de la Convención sobre los Derechos del Niño. Granada: GEU Editorial.

Jiménez, M. (2006): Menores inmigrantes o los vulnerables de la globalización. En Checa y Olmos, F. y Checa Olmos, J.C. (Eds.). Menores tras la frontera. Otra inmigración que aguarda. Barcelona: Icaria. 
Kohli, R. (2014). Proteger a los menores en situación de migración independiente. En Jiménez, A.S., Pantoja, A., Leiva, J.J. y Moreno, E. (Coords.). Infancia en contextos de riesgo. XXV Años de la Convención sobre los Derechos del Niño. Granada: GEU Editorial.

Konrad, M. y Santoja, V. (2005). Menores migrantes. De los puntos cardinales a la rosa de los vientos. Valencia: Promolibro.

Krmpotic, C. S. (1999): El concepto de Necesidad y políticas de Bienestar. Argentina: Espacio Digital.

Lacomba, J. (2008). Historia de las Migraciones Internacionales. Historia, geografía, análisis e interpretación. Madrid: Los libros de la Catarata.

Lahire, B. 2007. Infancia y adolescencia: de los tiempos de socialización sometidos a constricciones múltiples. Revista de Antropología Social, № 16, pp. 21-38.

López, O. y Aharchi, N. (2012). Discursos sobre la inmigración rrom (gitana) rumana en Barcelona. Revista Discurso y Sociedad, Vol. 6 (2), pp. 543-590.

Marcu, S. (2013). La movilidad transfronteriza de rumanos en España en tiempos de crisis. Revista Internacional de Sociología (RIS), No 71 (1), pp. 115-141. https://doi. org/10.3989/ris.2012.01.18

Martínez, P.C. (2006). El método de estudio de caso. Estrategia metodológica de la investigación científica. Revista pensamiento y gestión, № 20, pp. 165-193.

Massey, D.S. (1987). Understanding Mexican Migration to the United States. American Journal of Sociology, Vol. 92, № 6, pp. 1372-1403.

Matus, C. (1987). Adiós Señor Presidente. Planificación, Antiplanificación y Gobierno. Venezuela: POMAIRE.

Montenegro, S. (2005). La sociología de la sociedad del riesgo: Ulrich Beck y sus críticos. Pampa, Revista Interuniversitaria de Estudios Territoriales, №1, pp. 117-130. https:// doi.org/10.14409/pampa.v1i1.3122

Pajares, M. (2007). Inmigrantes del Este. Procesos migratorios de los rumanos. Barcelona: Icaria

Pozuelos, F.J., Rodríguez, F.P. y Travé, G. (2012). El enfoque interdisciplinar en la enseñanza universitaria y el aprendizaje basado en la investigación. Un estudio de caso en el marco de la formación. Revista de educación, № 357, pp. 561-585.

Proyecto Nido (2011). Proyecto Nido: Todos los niños/as tienen a una educación que asegure su integración social. Madrid: Fundación Imaginario Social.

Proyecto PUCAFREU. (2012). Promoting Unprotected Unaccompained Children 's Access to fundamental rights in the European Union. Bélgica, España, Francia, Italia y Rumania. (CNRS- Migrinter)/ Universidad de Poitiers (Francia).

Ryan, T. (1996). Risk Management and People with Mental Health Problems. H. Kemshall \& J.Pritchard (eds.), Good Practice in Risk Assessment and Risk Management, 1. Londres: Jessica Kigsley.

Selener, D. (1997). Participatory action research and social change. New York: Cornel University Participatory Action Research Network.

Rojo, T. (2010). Violencia juvenil y territorios urbanos. Revista Andaluza de Ciencias Sociales, Anduli, № 9, pp. 9-28. 
San Román, T. (2006). Integración social, aculturación y el valor de las relaciones interétnicas. En Checa y Olmos, F. Arjona, A. y Checa Olmos, J.C. (Eds.). Menores tras la frontera. Otra inmigración que aguarda. Barcelona: Icaria Editorial.

Shaw, E. (1999): A guide to the qualitative research process: Evidence from a small firm study. Qualitative market research: An International Journal, № 2 (2), pp. 59-70.

Sordé, T., Flecha, R. y Mircea, T. (2013). El pueblo gitano: una identidad global sin territorio. Scripta Nova, Revista Electrónica de Geografía y Ciencias Sociales, Vol. XVII, $\mathrm{N}^{\circ} 427(3)$.

Stake, R.E. (1998): Investigación con estudio de casos. Madrid: Morata.

Stevenson, O. (1999). Old People at Risk. Social Work, 36. Londres: Jessica Kingsley.

Suárez-Grimalt, L. (2017). Migración y movilidad social: una aproximación desde las estrategias de acumulación de activos de la población latinoamericana en España. Revista Española de Sociología, No 26 (3), pp.2-14. https://doi.org/10.22325/fes/res.2017.27

Subirats, J. (2005). Catorce puntos esenciales sobre evaluación de políticas públicas con especial referencia al caso de las políticas sociales. Ekonomiaz, $\mathrm{N}^{\circ} 60$, Vol. I.

Torres, A. (2013). Jitanos con Jota. Historia de vida Jitana. Sevilla: Copiarte.

Valderrama, R. (2013). Diagnóstico participativo con cartografía social. Innovaciones en metodología Investigación-Acción Participativa (IAP). Revista Andaluza de Ciencias Sociales, Anduli, No 12, pp. 53-66. https://doi.org/10.12795/anduli.2013.i12.03

Velaz de Medrano, C. (2002). Intervención educativa en sujetos con desadaptación social. Madrid: UNED.

Vergnano, C. (2014). Conexiones entre una agresión romófoba y un proyecto de regeneración urbanística en la periferia de Turín, Italia. Una aproximación crítica. Revista del área de estudios urbanos, Quid, №4, pp. 84-109.

Vidal, F. (2009). Pan y Rosas. Fundamentos de exclusión y empoderamiento. Madrid: Fundación FOESSA y CARITAS.

Viruela, R. (2016). La movilidad interna e internacional de los inmigrantes rumanos durante la crisis. Scripta Nova, Revista Electrónica de Geografía y Ciencias Sociales, № 536 , pp. 1-28.

Warner, F. (1992). Introduction. Risk: Analysis, Perception and Management Report of a Royal Society Study Group. Londres: Royal Society. 